\title{
Adhesion along metal-polymer interfaces during plastic deformation
}

\author{
R. van Tijum $\cdot$ W. P. Vellinga $\cdot$ J. Th. M. De Hosson
}

Received: 6 August 2005 / Accepted: 22 February 2006/ Published online: 20 January 2007

(C) Springer Science+Business Media, LLC 2007

\begin{abstract}
In this paper a numerical study is presented that concentrates on the influence of the interface roughness that develops during plastic deformation of a metal, on the work of adhesion and on the change of interface energy upon contact with a glassy polymer. The polymer coating is described with a constitutive law that mimics the behavior of Poly-Ethylene Terephthalate. It includes an elastic part, a yield stress, softening and hardening with increasing strains. For the interface between the metal and the polymer a mixed-mode (mode I and II) stress-separation law is applied that defines the interface energy and an interaction length scale. At the onset of deformation the surface of the substrate has a self-affine roughness characterized by the so-called Hurst exponent, a correlation length and an rms roughness amplitude, that evolves as a function of increasing strain. The findings are the following: the interface energy decreases until the strain at yield of the polymer coating. Interestingly, after yielding as the polymer starts to soften macroscopically, the decreasing average stress levels result in partial recovery of the interface energy at the interface. At higher strains, when macroscopic hardening develops the recovery of the interface stops and the interface energy decreases. The effect of coating thickness is discussed as well as the physical relevance of various model parameters.
\end{abstract}

R. van Tijum · W. P. Vellinga · J. Th. M. De Hosson ( $\square)$ Department of Applied Physics, The Netherlands Institute for Metals Research and Materials Science Center, University of Groningen, Nijenborgh 4, 9747 AG Groningen, The Netherlands

e-mail: j.t.m.de.hosson@rug.nl

\section{Introduction}

Polymer-coated metal sheets are rather recent products of steel manufacturers that are used in various applications in food and automotive industry. In the manufacturing process severe plastic deformation is used to obtain the final shapes of the end products. A drawback of plastic deformation is the intrinsic roughening of the surface of the metal caused by dislocation activity. This paper concentrates on the implications of the roughening process for the mechanical properties of the combined metal-polymer system. Clearly the subject is closely related to that of a large number of papers discussing the impact of roughness on the work of adhesion $W$, e.g [1-7]. However, this paper sets itself apart from earlier research because it emphasizes on: First, the evolution of roughness of the metal as a mechanical loading mechanism of a metal-polymer interface; second, the coupling between the metal substrate and the polymer coating using a stress-separation law; and third, the polymer behavior including yielding, softening and hardening. In the following these points are briefly discussed in the framework of the current understanding.

During uniaxial deformation, a metal surface roughens. A height-height correlation function $C(r, \varepsilon)$ of the interface may be defined as [8]

$$
C(r, \varepsilon)=\langle h(\vec{r}, \varepsilon) h(0, \varepsilon)\rangle,
$$

where $h(\vec{r}, \varepsilon)$ is the height of the surface at a strain $\varepsilon$ and at the position $\vec{r}$. It has been shown $[9,10]$ that for all strains $C(r, \varepsilon)$ of a surface of a uniaxially deformed metal can be approximated by: 
$C(r, \varepsilon) \approx w(\varepsilon)^{2} \exp \left[-(r / \xi(\varepsilon))^{2 H(\varepsilon)}\right]$.

Equation 2 describes the surface roughness in a statistical sense with three parameters: the rms roughness amplitude $w$ above a certain lateral correlation length $\xi$, and a Hurst exponent $H$ characterizing the self-affine geometry below $\xi$. For a uniaxially deforming metal these surface parameters are a function of the applied strain $\varepsilon$. From experiments parameterized fits for $w(\varepsilon), H(\varepsilon)$ and $\xi(\varepsilon)$ may be determined (see also $[9,11])$.

The topics addressed here are the work of adhesion $W$ between such a self-affine roughening metal-polymer system, the dependence of $W$ on the parameters $w$, $H$ and $\xi$, and the evolution of $W$ as a function of uniaxial strain $\varepsilon$.

Figure 1 illustrates the problem under consideration, and introduces a number of relevant quantities. It shows a metal and polymer in contact across an interface at zero strain, and also at a higher strain value. The area of contact increases upon straining, the average stress in the polymer increases, and roughness develops at the interface, which is accompanied by stress concentrations. These stress concentrations can lead to local delamination. Also, note the appearance of shear bands in the polymer, caused by the intrinsic softening that characterizes the post-yield deformation behavior of typical glassy polymers. The impact of all of these phenomena on the work of adhesion $W$ is the subject of this paper.

For a metal $(m)$ and a polymer $(p)$ coming into contact across an area $A_{0}$ the work of adhesion $W$ per unit area is defined as:

$W=G_{0}=\gamma_{m}+\gamma_{p}-2 \gamma_{m p}$

where $\gamma_{m}$ and $\gamma_{p}$ represent the surface energies and $\gamma_{p m}$ the interface energy. During uniaxial deformation at a

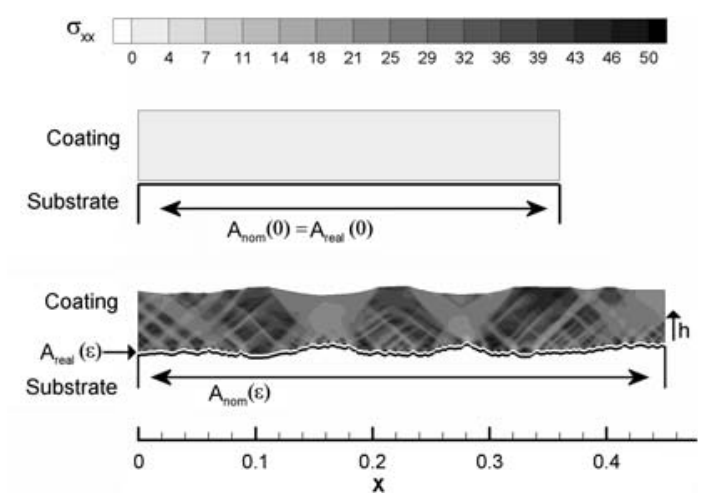

Fig. 1 Schematic representation of some model parameters at two stages in the deformation process $(\varepsilon=0$ and $\varepsilon>0)$ strain $\varepsilon$ the nominal (projected) contact area $A_{\text {nom }}$ is given by:

$A_{\text {nom }}(\varepsilon)=A_{0}(1+\varepsilon)$.

Due to roughening of the metal surface the real contact area $A_{\text {real }}$ at a strain $\varepsilon$ is larger than $A_{\text {nom }}(\varepsilon)$ and is given by

$A_{\text {real }}(\varepsilon)=\int d A(\vec{r})$

where the integral is taken over all positions $\vec{r}$ on the nominal surface.

The work of adhesion $w(\varepsilon)$ at a strain $\varepsilon$ can be approximated by:

$W(\varepsilon)=G(\varepsilon)-\frac{U_{E}(\varepsilon)}{A_{\text {nom }}(\varepsilon)}=\frac{\int G(\vec{r}) d A(\vec{r})}{A_{\text {nom }}}-\left(\frac{U_{E}^{h}(\varepsilon)+U_{E}^{\varepsilon}(\varepsilon)}{A_{\text {nom }}(\varepsilon)}\right)$

here $G(\varepsilon)$ represents all surface and interface terms and $U_{E}(\varepsilon)$ is the elastic energy stored in the bulk of the materials. The elastic energy is split in two terms:

First, $U_{E}^{\varepsilon}(\varepsilon)$ represents the average energy stored in a deformed block of material in case the surface does not roughen and may be approximated by

$U_{E}^{\varepsilon}(\varepsilon)=\frac{1}{2} d A_{\text {nom }} \frac{\sigma(\varepsilon)^{2}}{E_{\mathrm{pol}}}$,

where $d$ is the layer thickness, $E_{\mathrm{pol}}$ is the Young's modulus of the polymer. Due to the substantial difference of the elastic moduli the metal can be regarded as a rigid solid and is omitted from the energy balance.

Second, $U_{E}^{h}(\varepsilon)$ represents the elastic energy contribution of the roughening interface.

The term $G(\varepsilon)=\int G(\vec{r}) d A(\vec{r}) / A_{\text {nom }}$ takes into account the two competing effects of roughening on the interface energy, on the one hand an increase in real contact area, and on the other hand local delamination caused by the stresses acting on the interface.

So far, the interface was described in terms of energies only. In reality stresses are transferred across the interface until delamination occurs, see Fig. 1. The interface can be described in terms of a stress-separation law (e.g $\sigma(\Delta)$ with $\Delta$ the displacement at the interface. Traction and shear stresses, i.e. $\sigma_{n}$, and $\tau_{t}$ respectively, and resulting displacements $\left(\Delta_{n}, \Delta_{t}\right)$ may have components normal and tangential to the interface. The work of adhesion $W$ enters in a natural way as 
$W=G_{0}=\int_{0}^{\infty} \sigma \cdot d s$.

(short cut of: $\int \overline{\bar{\sigma}}_{i j} \cdot \vec{n}_{j} d \vec{r} / \int d A$ )

$G_{0}$ again contains all surface and interface terms. One can now also define

$G(\Delta)=G_{0}-\int_{0}^{\Delta} \sigma \cdot d s$.

In a physical sense $G(\Delta)$ represents the remaining work needed to separate two surfaces in the presence of a pre-applied stress. In the case of a roughening surface as considered here, the stresses at a certain position stem from the height differences, e.g. areas of the interface between two roughness peaks experience a local opening stress.

The separate contributions of interface and bulk to the work of adhesion and their dependence on the strain are a key issue. In the following our numerical approach is described that takes into account all the aforementioned features.

\section{Model description}

The numerical model describes the interface between steel and PET and it will capture the following three aspects: Polymer deformation; roughening of the metal surface; interaction between metal and polymer across the surface. The representation of the PET layer is a finite element model built from quadrilaterals representing a size of $1 \times 1 \mu \mathrm{m}^{2}[12,13]$. The initial length of the system is $2049 \mu \mathrm{m}$ and the initial thickness $h$ of the PET film is $60 \mu \mathrm{m}$ unless mentioned otherwise. In the calculations the 'metal' substrate is taken to be rigid. This is a reasonable approximation, since the elastic modulus of steel is typical of the order of $200 \mathrm{GPa}$ and therefore the thin polymer coating is expected to have little effect on its roughening behavior.

\section{Polymer constitutive behavior}

The constitutive behavior used for the polymer is described in [12] and the response and values of the parameters are given in the caption of Fig. 2. Here the curve is plotted as a function of the true strain $\varepsilon_{\text {true }}$, which is defined as $\varepsilon_{\text {true }}=\ln (1+\varepsilon)$. The regime of the polymer is purely elastic and after yielding the polymer starts to soften. In this regime shear bands occur as a result of localization. Eventually, hardening (onset just visible in the figure) will stop the localization.

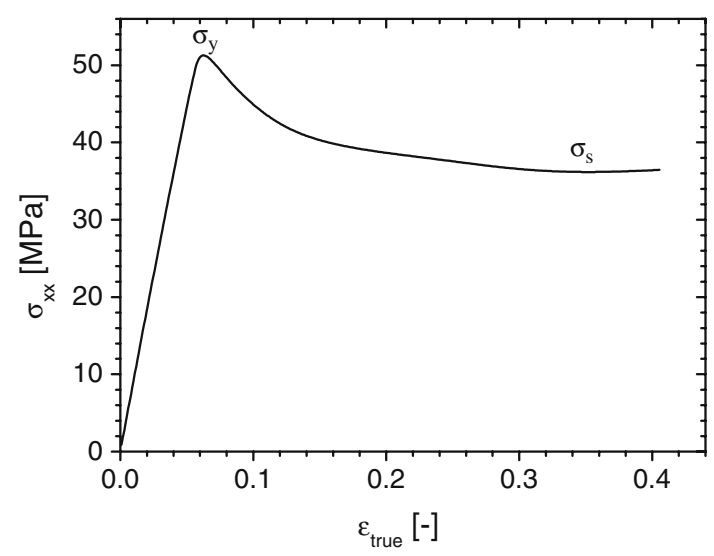

Fig. 2 Stress-strain relationship for PET, using the following values as defined in [12]: $E / s_{0}=7.76 ; s_{s S} / s_{0}=0.774 ; A s_{0} / T=91.99$; $h / s_{0}=3.90 ; \alpha=0.25 ; N=12.602 ; C_{R} / s_{0}=0.132$

Roughening of the metal surface

One of the simplifications in the approach proposed here is to parameterize the roughness evolution of a metal surface as a function of strain, and to assume that the roughness evolution at a polymer-metal interface is essentially identical to this because of the large difference in elastic moduli of typical metals $(\sim 100 \mathrm{~s}$ of GPa) and glassy polymers ( $1 \mathrm{GPa})$.

The surface morphology for as received rolled stainless steel (with an averaged grain size equal to $11.7 \mu \mathrm{m}$ and a plate thickness of $500 \mu \mathrm{m}$ ) was determined experimentally with confocal microscopy [9] during uniaxial tensile experiments and characterized by the following empirical relationships as a function of strain $\varepsilon$ :

$w(\varepsilon)=w_{\text {sat }}\left(1-\mathrm{e}^{-C_{1} \varepsilon}\right) \xi(\varepsilon)=\xi_{0}(1+\varepsilon)$,

with $w_{\text {sat }}=1.1 \mu \mathrm{m}, C_{1}=6.1$ and $\xi_{0}=35 \mu \mathrm{m}$. The Hurst exponent $H$ was found to be insensitive to strain and is taken to be constant $H(\varepsilon)=H_{0}=0.6$. The correlation length $\xi$ increases with the strain in the tensile direction. A qualitatively similar behavior was also found for other materials (Fe, Al, [10]). From experimental results least square fits to Eq. 10 were performed [9]. A recursive refinement algorithm [11] was used to simulate surfaces with the characteristics described by Eq. 10. A detailed example of the roughness evolution in the numerical model is displayed in [9], a few stages of which are apparent from Fig. 3.

Interaction between metal and polymer

In finite element models, stress-separation laws are commonly known as "cohesive zones" and different 
$\begin{array}{lllllllllllllll}10 & 12 & 14 & 16 & 19 & 21 & 23 & 25 & 27 & 29 & 31 & 34 & 36 & 38 & 40\end{array}$

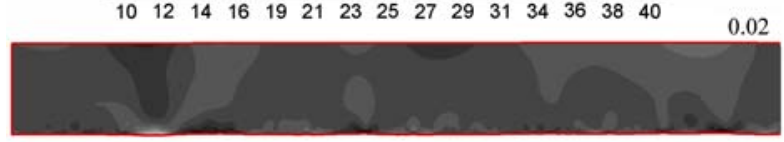

0.18

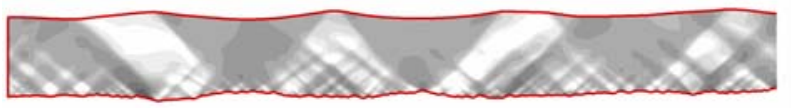

0.41

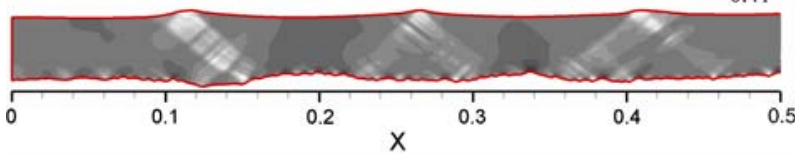

Fig. 3 Typical results of stress fields in the PET caused by strain induced roughening of the substrate, showing $\sigma_{x x}$ in the PET. Three cases are shown for in the elastic (top), softening (middle) and hardening (bottom) region of the polymer stress-strain curve

kinds have been discussed in the literature. In the numerical calculations presented here the interface was implemented as a rate-independent mixed-mode cohesive zone of the type described in [14]. This type defines coupled stress separation laws $\sigma_{n}\left(\Delta_{n}, \Delta_{t}\right)$ (tractions) and $\tau_{t}\left(\Delta_{n}, \Delta_{t}\right)$ (shear stresses) at the interface, with $\Delta_{n}$ and $\Delta_{t}$ coordinates normal and parallel to the interface, respectively. The distance $\Delta_{0}$ is defined as the point at which the opening stress $\sigma_{n}$ attains its maximum value $\sigma_{n}^{\max }$. Unless stated otherwise the parameters used for the cohesive zone in this paper are: normal-to-shear stress ratio $\sigma_{n}^{\max } / \tau_{t}^{\max }=0.75$, interface energy $G_{0}^{\mathrm{CZ}}=30 \mathrm{~J} / \mathrm{m}^{2}$, working distance $\Delta_{0}=300 \mathrm{~nm}$ and $\sigma_{n}^{\max }=36.8 \mathrm{MPa}$. To illustrate the key features of the cohesive zone $\sigma_{n}\left(\Delta_{n}, 0\right)$ is shown in Fig. 4.

For each interface element the surface area and stress state are calculated. The interface energy can be calculated analogous to Eq. 9 as:

$G_{i}^{\mathrm{CZ}}(\varepsilon)=G_{0}^{\mathrm{CZ}}-\int_{0}^{\Delta_{n}} \sigma_{i, n}^{\mathrm{CZ}}(\varepsilon) d n-\int_{0}^{\Delta_{t}} \tau_{i, t}^{\mathrm{CZ}}(\varepsilon) d t$,

where $G_{0}^{\mathrm{CZ}}$ refers to $G_{0}$ of the cohesive zone (see Eq. 8), $\sigma_{i, n}^{\mathrm{CZ}}$ is the stress normal and $\tau_{i, t}^{\mathrm{CZ}}$ the stress parallel to the interface element $i$. In this work we are interested in $w(\varepsilon)$ and therefore in $G(\varepsilon)$ and $U(\varepsilon)$. In the context of the numerical model we define $G(\varepsilon)$ as follows:

$G^{\mathrm{CZ}}(\varepsilon)=\frac{\sum_{i=1}^{N} G_{i}^{\mathrm{CZ}}(\varepsilon) A_{i}(\varepsilon)}{A_{\mathrm{nom}}}$

with $i$ running over the discrete elements in the model, $A_{i}(\varepsilon)$ and $G_{i}^{\mathrm{CZ}}(\varepsilon)$ are the surface area and the interface energy of the i-th element, respectively.
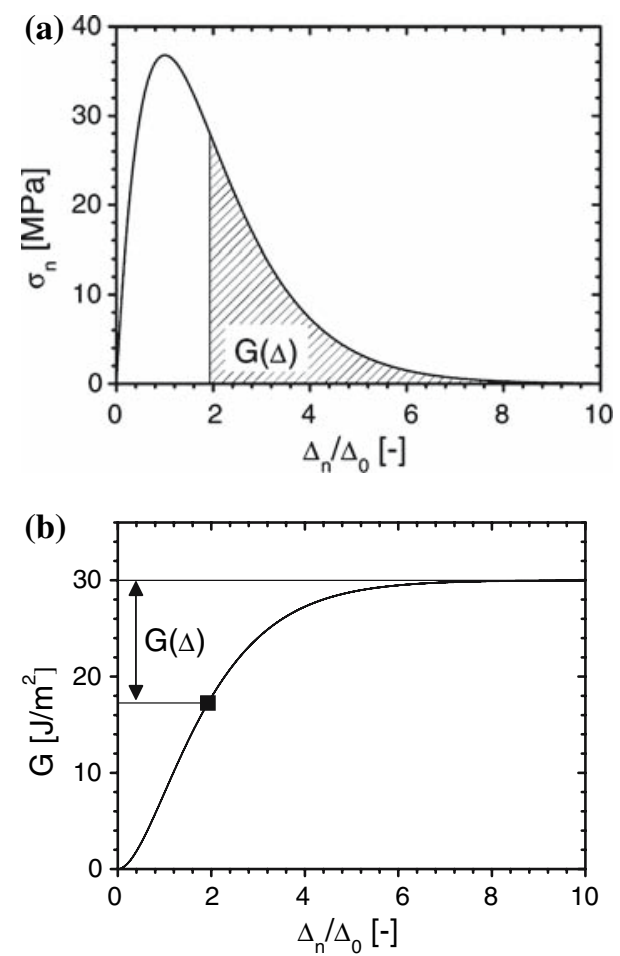

Fig. 4 Characteristics of the cohesive zone (a) $\sigma_{n}\left(\Delta_{n}, 0\right)$ plotted as function of $\Delta_{n} / \Delta_{0}$ (b) $G\left(\Delta_{n}\right)=\int_{\Delta_{n}}^{\infty} \sigma_{n}(n, 0) d n$ plotted as function of $\Delta_{n} / \Delta_{0}$

Since all energies in the following are derived from the numerical model the superscript $\mathrm{CZ}$ will be dropped.

Simulation of deformation

In the calculations the composite is loaded in uniaxial plane strain up to strains of $50 \%$ (in steps of $0.1 \%$ ). At each step the following boundary conditions (see Fig. 5) are imposed on the PET and the cohesive zone. At the side of the PET at $x=0$ displacements along $x$ are imposed while displacements along $y$ are free. Similar boundary conditions are applied at $x=L(\varepsilon)$. Along the interface displacements in the substrate are constrained in all directions. Displacements in the polymer are not restricted and coupled to those in the substrate by the stress-separation laws incorporated in the cohesive zone. At each strain step the positions of the substrate nodes are updated in $x$

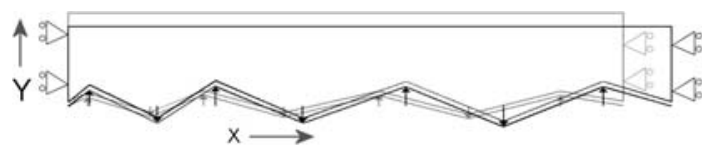

Fig. 5 Schematic representation of the boundary conditions at low strain (gray) and higher strain (black) 
(to reflect the increase in strain) and $y$ (to reflect the increase in roughness).

\section{Results}

A typical result of the calculation is shown in Fig. 3. The figure shows $\sigma_{x x}$ distribution in the PET at strains for three different stages depicted in Fig. 2: elastic I, softening II, and hardening III. Clearly visible are localized shear bands and roughening of the polymer surface during the softening and hardening regime.

A series of calculations was carried out in which the parameters describing the roughness and the parameters describing the cohesive zone were varied. For these calculations $w(\varepsilon)$ and $G(\varepsilon)$ were studied and are discussed below.

\section{General characteristics of $G(\varepsilon)$}

All graphs of $G(\varepsilon)$ display a number of characteristic features. These will be discussed using Fig. 6 that shows the dependence of $G(\varepsilon)$ on $\varepsilon_{\text {true }}$. (Note that in the figures $G(\varepsilon) / G_{0}$ has been plotted, rather than $G(\varepsilon)$. Three stages can be distinguished. $G(\varepsilon)$ decreases initially, approximately up to $\sigma_{y}$ (stage I) and then starts to increase (stage II), after which it passes through a maximum and decreases again (stage III). Depending on $w_{\text {sat }}$ the maximum may be either below or above $G_{0}$.

The physical interpretation of the three stages is as follows: in stage I, "delamination" due to the loading of interface by the coating dominates and $G(\varepsilon)$ decreases. In stage II the area increase due to roughening dominates because during this stage the stress in polymer is limited by $\sigma_{y}$. Finally, in stage III the polymer starts to work harden, the load at the interface increases and delamination proceeds. This effect levels

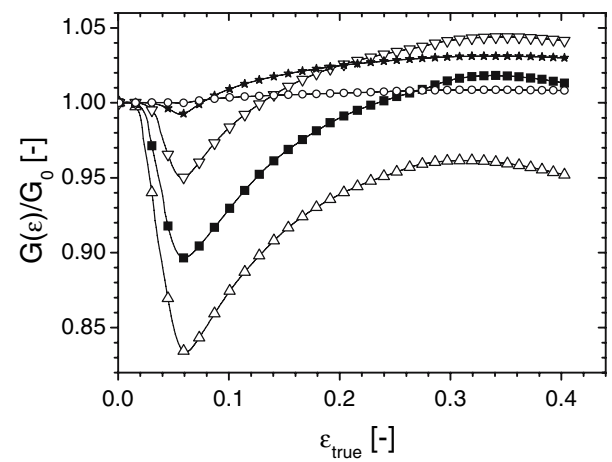

Fig. 6 Normalized interface energy $G(\varepsilon) / G_{0}$ as function of $\varepsilon$ for different values of $w_{\text {sat }}: \bigcirc w_{\text {sat }}=1.1 \mu \mathrm{m} ; \quad \star w_{\text {sat }}=2.2 \mu \mathrm{m}$; $\nabla w_{\text {sat }}=3.3 \mu \mathrm{m} ; \boldsymbol{\square} w_{\text {sat }}=4.4 \mu \mathrm{m}$ and $\Delta w_{\text {sat }}=5.5 \mu \mathrm{m}$ off because of the saturation of the roughness amplitude with increasing strain. The interpretation of the three stages is confirmed in Fig. $7 a$ and $b$ showing the effects of delamination and area increase respectively.

Here it is useful to split the effect of the roughness and the increase in surface area (as shown in Fig. 7a and $b)$ :

$G(\varepsilon)=G_{0} \frac{A_{\text {real }}(\varepsilon)}{A_{\text {nom }}(\varepsilon)}+\Delta G(\varepsilon)$,

where the effect of the roughness on the change of interface energy upon contact is

$\Delta G(\varepsilon)=-\frac{\sum_{i=1}^{N} G_{i}(\varepsilon) A_{i}(\varepsilon)}{\sum_{i=1}^{N} A_{i}(\varepsilon)}$

and the surface area increase effect is

$\frac{A_{\text {real }}(\varepsilon)}{A_{\text {nom }}(\varepsilon)}=\frac{\sum_{i=1}^{N} A_{i}(\varepsilon)}{A_{\text {nom }}(\varepsilon)}$.

Figure 7 a shows the reduction in interface energy as indicated in Eq. 14. This effectively removes the effect of increasing area and gives an indication of the decrease in $G(\varepsilon)$ caused by the loading of the interface. At the onset $\Delta G(\varepsilon)$ decreases rapidly (approximately
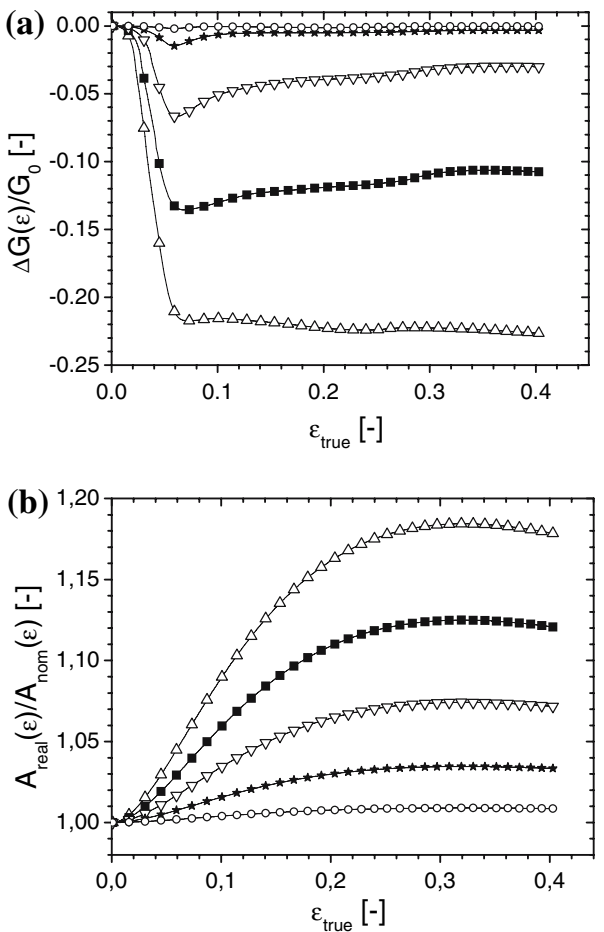

Fig. 7 Normalized interface energy $G(\varepsilon) / G_{0}$ (same data as in Fig. 6) separated into the contribution (a) $(\varepsilon) / G_{0}$ and (b) $A_{\text {real }}(\varepsilon) / A_{\text {nom }}(\varepsilon)$ 
linearly with $w^{2}$ ). At the yield point of the polymer the rate of decrease of $\Delta G(\varepsilon)$ reduces considerably, and in fact for low $w_{\text {sat }}$ the interface partly recovers due to the softening of the polymer layer, and the reduced stresses acting on the interface as a cause of that.

Figure $7 \mathrm{~b}$ shows $A_{\text {real }}(\varepsilon) / A_{\text {nom }}$ which exhibits a monotonous increase due to roughening up to a maximum where the increase in nominal area starts dominating the effects of the increase of $w(\varepsilon)$.

$G(\varepsilon)$ as function of $w, H$ and $\xi$

In the previous section the general behavior of $G(\varepsilon)$ was illustrated, and curves at different $w_{\text {sat }}$ were presented. The situation remains roughly the same if we change other parameters describing the self-affinity, the correlation length $\xi$ and the Hurst exponent $H$. A low value of $H$ results in rapid fluctuation on a short length scale, and this leads to a larger decrease in $G(\varepsilon)$ at low strains (Fig. 8a) and to a continuing delamination above the yield point. However, the overall effect is still compensated by the faster increase in surface area (Fig. 8b).

$W(\varepsilon)$ as a function of layer thickness

We note that the effects shown in the previous section do not take into account the elastic energy stored in the layer. Figure 9 shows $G(\varepsilon)$, similar to Figs. 6 and $7 \mathrm{~b}$ for layers of different thickness. For all layer thickness the behavior of $G$ is qualitatively similar. Figure 10 shows $W(\varepsilon)$ up to the strain at yielding. $W(\varepsilon)$ equal to zero indicates that the interface becomes metastable to fracture. Clearly, $U_{E}(\varepsilon) / A_{\text {nom }}(\varepsilon)$ dominates $W(\varepsilon)$. For the $10 \mu \mathrm{m}$ coating $W(\varepsilon)$ decreases to a value of about 0.6 at the yield strain, indicating that this coating is stable against delamination. $\frac{U_{E}(\varepsilon)}{A_{\text {nom }}(\varepsilon)}$ increases linearly with $d$, and for the coatings of $30 \mu \mathrm{m}$ and of $60 \mu \mathrm{m}$ the energy $W(\varepsilon)$ turns out to be smaller than zero and therefore these situations are metastable.

\section{Discussion}

A relevant point of discussion is the influence of the cohesive zone on the results. In Fig. 11 the results for three different parameter sets describing the cohesive zone (in the inset) are shown. Regardless of the parameter values of the cohesive zone the same qualitative behavior of the $w(\varepsilon)$ is found, i.e. weakening, recovery and renewed weakening.

Another interesting aspect is the relation to analytical results relating $G(\varepsilon)$ to the roughness of interfaces.
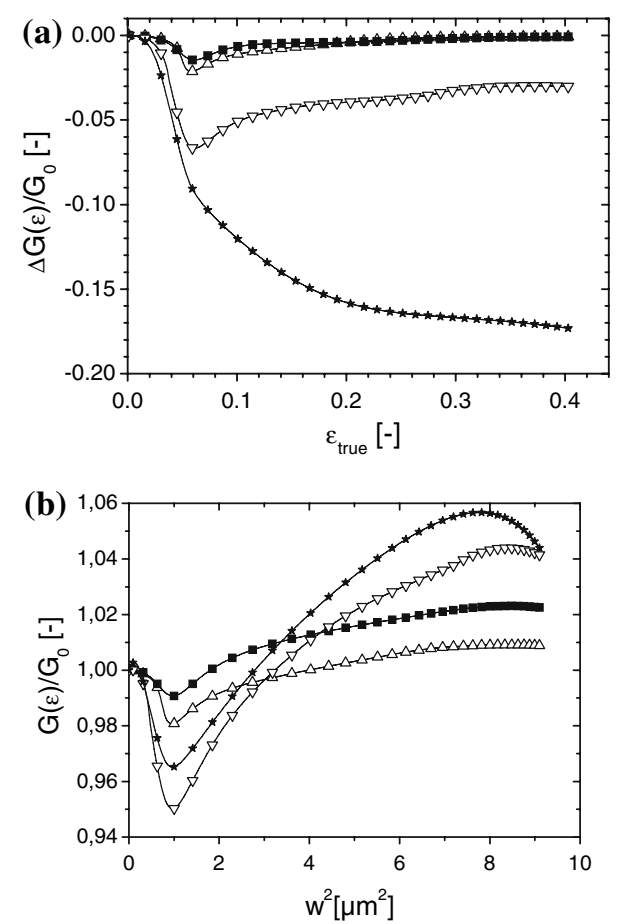

Fig. 8 (a) $\Delta G(\varepsilon) / G_{0}$ as a function of $\varepsilon$, and (b) $G(\varepsilon) / G_{0}$ as a function of $w^{2}$. For all curves $w_{\text {sat }}=3.3 \mu \mathrm{m}$. Hurst exponents $H: \star \mathrm{H}=0.4 ; \nabla \mathrm{H}=0.6 ; \Delta \mathrm{H}=0.8$ and $\mathrm{H}=1.0$

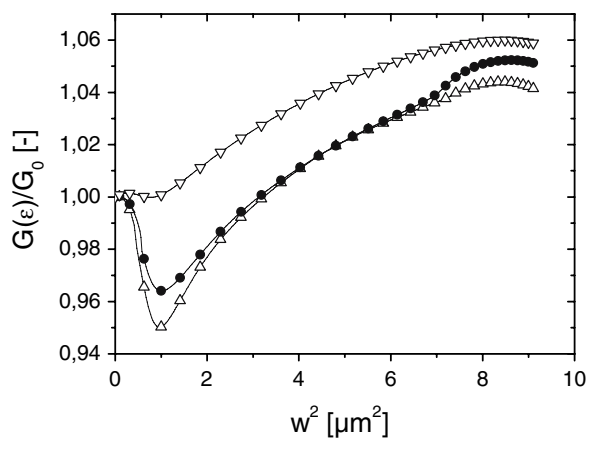

Fig. $9 G(\varepsilon) / G_{0}$ as a function of $w^{2}$. For all curves $w_{\text {sat }}=3.3 \mu \mathrm{m}$. Thickness $h: \nabla h=10 \mu \mathrm{m} ; \bullet h=30 \mu \mathrm{m}$ and $\Delta h=60 \mu \mathrm{m}$

In the literature analytical studies have been reported for situations $[15,16]$ in which a flat elastic body is brought into perfect contact with a rigid rough body (see appendix A for a brief overview of these treatments).

The physical picture emerging from these analytical results is the following: Roughness increases the real contact area at the interface and this effect contributes to an increase of the change of interface energy upon contact. On the other hand for complete contact to occur elastic energy is stored in the material and this contributes to a decrease in interface energy. Depend- 


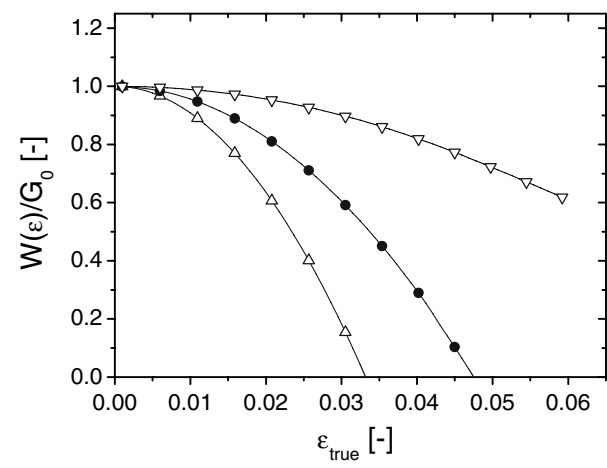

Fig. 10 Work of adhesion $W$ of the system for different coating thickness $h: \nabla h=10 \mu \mathrm{m} ; \bullet h=30 \mu \mathrm{m}$ and $\Delta h=60 \mu \mathrm{m}$ (same dataset as Fig. 9)

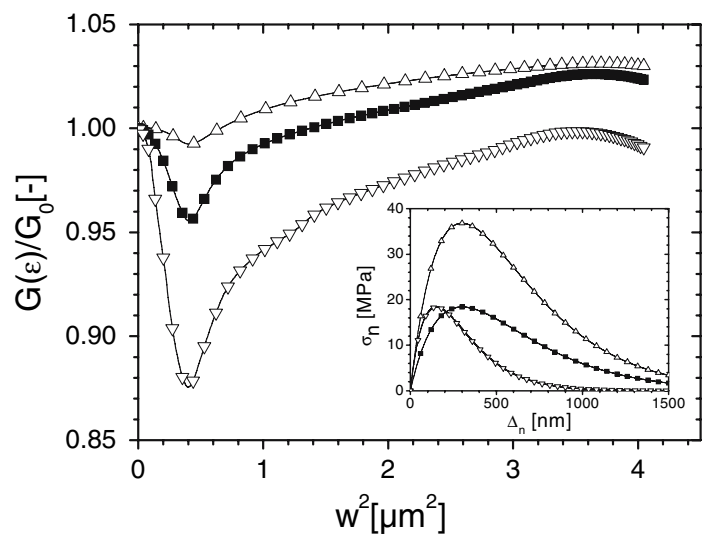

Fig. 11 Normalized effective interface energy $G(\varepsilon) / G_{0}$ as a function of the squared rms roughness $\left(w_{\text {sat }}=2.2\right)$ for different $G_{0}$ and working distance $\Delta_{0}: \nabla z_{0}=150 \mathrm{~nm}, \sigma_{\text {max }}^{n}=18.4 \mathrm{MPa}$ and $G_{0}=7.5 \mathrm{~J} / \mathrm{m}^{2} ; \quad z_{0}=300 \mathrm{~nm}, \sigma_{\max }^{n}=18.4 \mathrm{MPa}$ and $G_{0}$ $=15 \mathrm{~J} / \mathrm{m}^{2} ; \Delta z_{0}=300 \mathrm{~nm}, \sigma_{\max }^{n}=36.8 \mathrm{MPa}$ and $G_{0}=30 \mathrm{~J} / \mathrm{m}^{2}$

ing on the properties of interface, substrate and polymer (geometric as well as elastic) one of these two effects dominates and in fact a critical modulus $E_{\mathrm{c}}$ can be defined for the polymer layer that separates these two regimes [15]. For $E_{\mathrm{c}}$ it is found that

$E_{\mathrm{c}}=\frac{4 \pi}{\xi(\varepsilon)}\left(1-v^{2}\right) G_{0} g\left(H_{0}\right) / f\left(H_{0}\right)$

with

$f\left(H_{0}\right)=\frac{H_{0}}{1-2 H_{0}}\left[\left(\frac{\xi_{0}}{r_{\min }}\right)^{1-2 H_{0}}-1\right]$

and

$g\left(H_{0}\right)=\frac{H_{0}}{2\left(1-H_{0}\right)}\left[\left(\frac{\xi_{0}}{r_{\text {min }}}\right)^{2\left(1-H_{0}\right)}-1\right]$.
Here $r_{\text {min }}$ represents the smallest length scale in the system (in our case $1 \mu \mathrm{m}$ ). In the case of PET and steel and for a substrate geometry typical for the ones discussed here we find $E_{\mathrm{c}} \sim 70 \mathrm{MPa}$ which means that $E_{\mathrm{PET}} \gg E_{\mathrm{c}}$. So, based on the analytical approach the interfaces are expected to show a decrease in interface energy for increasing roughness. An expression for $G(\varepsilon) / G_{0}$ is given in [15] (see Appendix A for a derivation and a description of the assumptions):

$$
\begin{aligned}
\frac{G(\varepsilon)}{G_{0}}= & \int_{0}^{\infty}\left(1+\frac{8 \pi^{2}}{\xi(\varepsilon)^{2}} w(\varepsilon)^{2} g\left(H_{0}\right) x\right)^{\frac{1}{2}} \mathrm{e}^{-x} d x \\
& -\frac{E}{E_{\mathrm{c}}} \frac{4 \pi^{2}}{\xi(\varepsilon)^{2}} w(\varepsilon)^{2} g\left(H_{0}\right)
\end{aligned}
$$

In Fig. 12 a comparison is made between this analytical solution and a number of results from the numerical simulations (Fig. 6). To compare both cases it is necessary to introduce an effective modulus $E$ that reflects the elastic properties of both the cohesive zone and the polymer coating. In the graph a value of $118 \mathrm{MPa}$ is used.

The figure shows that the analytic result (indicated by a drawn line) predicts a monotonous linear decrease of $G(\varepsilon) / G_{0}$ as a function of $w(\varepsilon)^{2}$. We note that the existence of an enveloping curve (indicated by a dashdot line in the figure) may be inferred from the numerical simulations (also shown in Fig. 11). This shows that as long as the PET is in the elastic regime the interface energy depends only on $w(\varepsilon)^{2}$ which is in qualitative accordance with the analytical results. A difference in this respect is the occurrence of a nonlinear regime at low strains for the numerical solutions which is due to the description of the interface with a

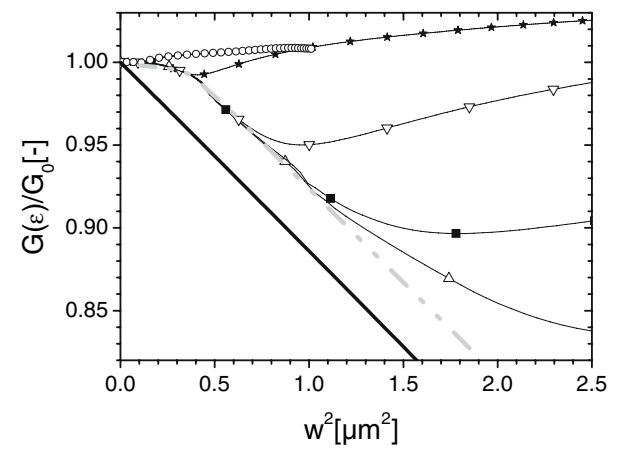

Fig. 12 Comparison of the results of Eq. 17 (with $E=118 \mathrm{MPa}$ ) indicated with the drawn line and numerical results $\bigcirc w_{\text {sat }}=1.1 \mu \mathrm{m} ; \star w_{\text {sat }}=2.2 \mu \mathrm{m} ; \quad \nabla w_{\text {sat }}=3.3 \mu \mathrm{m} ; \quad \square w_{\text {sat }}=$ $4.4 \mu \mathrm{m}$ and $\Delta w_{\text {sat }}=5.5 \mu \mathrm{m}$. An enveloping curve (dash-dot) accentuate the response of the numerical model in case the PET behaves elastically 
stress-separation law with a certain working distance. Decreasing the interaction distance for the cohesive zone will lead to a closer correspondence with the analytical result.

Deviation from the enveloping curve occurs for all numerical calculations as soon as the average strain in the PET reaches the yield strain. The value of $w(\varepsilon)^{2}$ at which this occurs depends on $w_{\text {sat }}$. It can be seen that for small $w_{\text {sat }}$ the deviations from the envelope curve occur for very low $w(\varepsilon)^{2}$. The softening that occurs in the PET above the yield strain leads to an increase or partial recovery of $G(\varepsilon) / G_{0}$, a behavior that differs drastically from what is expected from the analytical result.

\section{Conclusion}

The following generic picture emerges that describes the energetics of a strained ductile glassy polymer layer with a roughening interface:

At the interface local delamination leading to a decrease in adhered area competes with roughening that leads to an increase in adhered area.

A decrease of the interface energy occurs in the regime where the polymer deforms elastically, a (partial) recovery occurs during the softening phase of the polymer followed by a renewed decrease during the hardening phase of the polymer coating.

For layers of practical thickness the elastic energy stored in the polymer coating by straining at the yield stress dominates the work of adhesion and the stability of the interface against delamination.

Acknowledgements This work was financially supported by the Netherlands Technology Foundation STW (project number GTF.4901).

\section{Appendix A}

In the literature analytical studies have been reported for situations $[15,16]$ in which a flat elastic body is brought into perfect contact with a rigid rough body. Using the definitions in Eqs. 3-7 and accounting for the self-affine character of the surface it can be shown that the results in $[15,16]$ are equivalent to:

$U_{E}^{h}(\varepsilon)-G_{0} A_{\text {real }}(\varepsilon)=-G(\varepsilon) A_{\text {nom }}(\varepsilon)$.

For $U_{E}^{h}(\varepsilon)$, the elastic energy in the polymer due to the surface roughness only, it is found that $[15,16]$ :

$U_{E}^{h}(\varepsilon)=A_{\text {nom }}(\varepsilon) \frac{E}{4\left(1-v^{2}\right)} \int q C(q, \varepsilon) d^{2} q$, where $q$ is defined as $\frac{2 \pi}{r}$ and $C(q, \varepsilon)$ is the Fourier transform of the substrate height-height correlation function $C(r, \varepsilon)$ defined in Eq. 1 [17-20]. For the term $G_{0} A_{\text {real }}(\varepsilon)$ it is found that

$G_{0} A_{\text {real }}(\varepsilon)=G_{0} A_{\text {nom }}(\varepsilon)\left(1+\frac{1}{2} \int q^{2} C(q, \varepsilon) d^{2} q\right)$.

For $C(r, \varepsilon)$ with scaling properties given by Eq. 2, $C(q, \varepsilon)$ scales as $C(q, \varepsilon) \propto \mathrm{q}^{-2-2 \mathrm{H}}$ if $q \xi \gg 1$, and as $C(q, \varepsilon) \propto \mathrm{w}^{2}$ if $q \xi \ll 1$. Using the latter relation together with Eqs. 19, 20 and 21 results in Eq. 18. These results hold when a number of criteria are met, most importantly $|\nabla h| \ll 1$ and the surfaces should stay in complete contact.

\section{References}

1. Hertz H (1882) J Reine Angew Math 92:156

2. Archard JF (1957) Proc R Soc London Ser A243:190

3. Greenwood JA, Williamson JBP (1960) Proc R Soc London Ser A295:300

4. Johnson KL (1985) Contact Mechanics. Cambridge University Press, Cambridge

5. Bush AW, Gibson RD, Thomas TR (1975) Wear 35:87

6. Persson BNJ, Bucher F, Chiaia B (2002) Phys Rev B 65(18): 184106

7. Hyun S, Pei L, Molinari JF, Robbins MO (2004) Phys Rev E 70(2):026117

8. Zhao Y, Wang GC, Lu TM (2001) Characterization of amorphous and crystalline rough surface. Principles and applications. Academic Press

9. van Tijum R, Vellinga WP, De Hosson JThM (2005) Linking length scales in the mechanical behavior of materials. In: Rudd RE, Balk TJ, Windl W, Bernstein N (eds) MRS Proceedings Volume 882E, EE3.2

10. Wouters O, Vellinga WP, van Tijum R and De Hosson JThM (2005) Acta Mater 53:4043-4050

11. van Tijum R, De Hosson JThM (2005) J Mater Sci 40:3503 DOI: $10.1007 / \mathrm{s} 10853-005-0414-4$

12. Van der Giessen E (1997) Eur J Mech, A/Solids 16(special issue): 87

13. Abdul-Baqi A, Van der Giessen E (2002) Int J Solid Struct 39(6): 1427

14. Xu XP, Needleman A (1993) Model Simul Mater Sci Eng 1:111

15. Persson BNJ, Tosatti E (2001) J Chem Phys 115:5597

16. Palasantzas G, De Hosson JThM (2003) Phys Rev E 67:021604/1-6

17. Krim J, Palasantzas G (1995) Int J Mod Phys B 9:599

18. Meakin P (1998) Fractals, scaling, and growth far from equilibrium. Cambridge University Press, Cambridge

19. Sinha SK, Sirota EB, Garoff S, Stanley HB (1988) Phys Rev B 38:2297

20. Yang HN, Lu TM (1995) Phys Rev E 51:2479 